\title{
Temporal precedence of emotion over attention modulations in the lateral amygdala: Intracranial ERP evidence from a patient with temporal lobe epilepsy
}

\author{
GiLles Pourtois \\ Ghent University, Ghent, Belgium \\ Laurent Spinelli and Margitta Seeck \\ University Hospital, Geneva, Switzerland \\ and University of Geneva, Geneva, Switzerland \\ AND \\ Patrik Vuilleumier \\ University of Geneva, Geneva, Switzerland
}

\begin{abstract}
Previous fMRI studies have reported mixed evidence for the influence of selective attention on amygdala responses to emotional stimuli, with some studies showing "automatic" emotional effects to threat-related stimuli without attention (or even without awareness), but other studies showing a gating of amygdala activity by selective attention with no response to unattended stimuli. We recorded intracranial local field potentials from the intact left lateral amygdala in a human patient prior to surgery for epilepsy and tested, with a millisecond time resolution, for neural responses to fearful faces appearing at either task-relevant or task-irrelevant locations. Our results revealed an early emotional effect in the amygdala arising prior to, and independently of, attentional modulation. However, at a later latency, we found a significant modulation of the differential emotional response when attention was directed toward or away from fearful faces. These results suggest separate influences of emotion and attention on amygdala activation and may help reconcile previous discrepancies concerning the relative responsiveness of the human amygdala to emotional and attentional factors.
\end{abstract}

The rapid detection of threat is crucial for the organism's survival. Converging evidence from behavioral (see Anderson, 2005; Ohman \& Mineka, 2001), neurophysiological (LeDoux, 1996), neuropsychological (Anderson \& Phelps, 2001; Vuilleumier \& Schwartz, 2001) and brain-imaging (Dolan \& Vuilleumier, 2003; Vuilleumier, 2005; Vuilleumier, Armony, Driver, \& Dolan, 2001) studies lends support to the hypothesis that the human brain is equipped with specific mechanisms to swiftly appraise threat-related stimuli in the environment and engage attentional resources toward them so as to allow adaptive behaviors. These mechanisms are thought to be crucially dependent on the amygdala function and to subserve a distinct process of emotional attention (Vuilleumier, 2005), affording the capture of attention by unattended but emotionally relevant stimuli through neural pathways that are separate from those controlling voluntary visuospatial attention. The amygdala is known to play a predominant role in emotional processing and learning (see Phelps, 2006). Moreover, lesions of the amygdala have been shown not only to impair fear recognition in humans (Adolphs, Tranel, Damasio, \& Damasio, 1994, 1995; Calder, Lawrence, \& Young, 2001), but also to reduce threat-related activations in remote brain regions of the inferotemporal and parietal cortex (Vuilleumier, Richardson, Armony, Driver, \& Dolan, 2004), which are presumably subserving mechanisms of emotional attention via feedback connections received from the amygdala (Amaral, Behniea, \& Kelly, 2003; Sabatinelli, Bradley, Fitzsimmons, \& Lang, 2005; Surguladze et al., 2003; Vuilleumier \& Driver, 2007).

Consistent with such a role for efficient threat detection, the amygdala response to emotional stimuli has been found to arise automatically, without explicit attention to the stimuli or, sometimes, even without awareness (Anderson \& Phelps, 2001; Dolan \& Vuilleumier, 2003; Jiang \& He, 2006; LeDoux, 1996; Morris, DeGelder, Weiskrantz, \& Dolan, 2001; Morris, Ohman, \& Dolan, 1999; Ohman \& Mineka, 2001; Pasley, Mayes, \& Schultz, 2004; Whalen et al., 1998; Williams, Morris, McGlone, Abbott, \& Mat- 
tingley, 2004). However, the current evidence still remains highly controversial as to whether emotional processing in the amygdala is dependent on selective attention (Pessoa, McKenna, Gutierrez, \& Ungerleider, 2002). Conflicting results have been observed by some fMRI studies that systematically manipulated attention to emotional stimuli, some suggesting that the amygdala may activate to fearful faces both within or outside the current attentional focus (Anderson, Christoff, Panitz, De Rosa, \& Gabrieli, 2003; Pasley et al., 2004; Vuilleumier et al., 2001; Vuilleumier et al., 2004; Williams et al., 2004), and others reporting abolished responses for unattended or masked threatrelated stimuli (Pessoa, Japee, Sturman, \& Ungerleider, 2006; Pessoa, McKenna, et al., 2002; Pessoa, Padmala, $\&$ Morland, 2005). Still other studies have reported decreased responses to threat stimuli but increased responses to neutral or positive stimuli (Silvert et al., 2007; Williams, McGlone, Abbott, \& Mattingley, 2005). However, given its sluggish temporal resolution (Logothetis, Pauls, Augath, Trinath, \& Oeltermann, 2001), BOLD fMRI cannot unequivocally establish whether emotional and attentional effects actually occur in the amygdala but during distinct time periods, as predicted by models postulating rapid inputs to the amygdala prior to full attention (see Bar et al., 2001; Cowey \& Stoerig, 1991; LeDoux, 1996; Morris et al., 1999; Vuilleumier, 2005). Thus, on the basis of the current evidence, a possible prediction (Vuilleumier, 2005) could be that an early stimulus-driven response to emotional information might take place in the amygdala irrespective of attentional control (see also Dolan \& Vuilleumier, 2003; Vuilleumier, Armony, Driver, \& Dolan, 2003; Whalen et al., 2004), whereas selective attention might modulate the level of amygdala activation (and hence, gate the processing of threat-related stimuli) during a later time period, consistent with the effects of top-down regulatory influences exerted on visual pathways by higher level cortical regions involved in attentional control, such as the frontoparietal network (see also Armony \& Dolan, 2002; Krolak-Salmon, Henaff, Vighetto, Bertrand, \& Mauguiere, 2004; Pourtois, Schwartz, Seghier, Lazeyras, \& Vuilleumier, 2006; Vuilleumier \& Driver, 2007).

Existing data are still scarce regarding the time course of activation to threat-related stimuli in the human amygdala, in contrast to numerous neurophysiological studies that have focused on this structure in nonhuman primates (Amaral et al., 2003). Several neurophysiological studies have contributed to better characterize some response properties of the amygdala in nonhuman primates - most notably, by recording early category-selective responses to faces (see Hoffman, Gothard, Schmid, \& Logothetis, 2007; Leonard, Rolls, Wilson, \& Baylis, 1985; Nakamura, Mikami, \& Kubota, 1992) and by showing its ubiquitous involvement in aversive fear conditioning (LeDoux, 1996; Phelps \& LeDoux, 2005; Samson \& Pare, 2006). By contrast, only a few human studies have used intracranial recordings to examine the time course of activation of the human amygdala to visual threat-related stimuli, such as fearful faces (see Fried, MacDonald, \& Wilson, 1997; Halgren et al., 1994; Krolak-Salmon et al., 2004). Some of these studies looked at the spiking activity of single neurons and disclosed highly circumscribed stimulusdriven category-selective responses in the amygdala (as well as the hippocampus) to various combinations of facial expressions and identities (Fried et al., 1997; see also Kreiman, Koch, \& Fried, 2000), whereas other studies recorded intracranial local field potentials (iLFPs) to demonstrate that the human amygdala could differentiate between neutral and fearful faces after $200 \mathrm{msec}$ post stimulus onset (Krolak-Salmon et al., 2004).

It is noteworthy that Krolak-Salmon et al. (2004) also reported that an early differential neural activity in the amygdala around $200 \mathrm{msec}$ arose for fearful faces, relative to happy, neutral or disgusted ones, but only when the patients were asked to attend to the facial expression content (i.e., to count faces expressing surprise), not when the patients performed a gender discrimination task (i.e., to count male or female faces). This dissociation suggests a task-dependent modulation of this relatively early threat-related processing in the amygdala. However, these results contrast with those of several fMRI studies showing reliable activation to fearful faces when healthy observers processed nonemotional faces in a task involving gender discrimination (Morris et al., 1996; Phillips et al., 2001; Vuilleumier et al., 2003), including studies that directly compared explicit and implicit processing of emotion expressions (Critchley et al., 2000). Furthermore, this earlier intracranial study (Krolak-Salmon et al., 2004) did not assess the effects of emotional expressions (fearful vs. neutral) and selective attention (spatially attended vs. unattended faces) within the same task (see Vuilleumier et al., 2001) so as to test whether distinct effects of emotion and attention could be detected in the amygdala, or whether, alternatively, attentional effects precluded all (including early) emotional effects in this region. Only task demands were manipulated in this study (Krolak-Salmon et al., 2004). Because the patients' performance was worse on the facial expression task ( $88 \%$ correct) than on the gender discrimination task $(99 \%$ correct) (Krolak-Salmon et al., 2004) this factor could at least partly contribute to the reported difference in amygdala responses between these two tasks (see also Pessoa, McKenna, et al., 2002). Hence, it remains unclear whether emotional and attentional effects may actually occur in the same amygdala region, but during distinct time periods (as predicted by some models; see, e.g., Vuilleumier, 2005), or instead, whether attentional control can merely suppress the early emotional response of the amygdala under some circumstances (in accord with a selective gating of emotional processing by selective attention in this brain structure; see, e.g., Pessoa, Kastner, \& Ungerleider, 2002; Pessoa, McKenna, et al., 2002).

We had the opportunity to address this question by recording iLFPs, with a millisecond time resolution (Logothetis et al., 2001), from face-selective contacts in the left lateral amygdala of a unique human patient with medically refractory epilepsy (see Figure 1A). Patient C.T. was selected for our study because he had structurally intact left temporal lobe and amygdala (see the case description below). Recordings were performed during the same task as that used in several previous fMRI studies (Bentley, Vuilleumier, Thiel, Driver, \& Dolan, 2003; Bishop, Dun- 
A

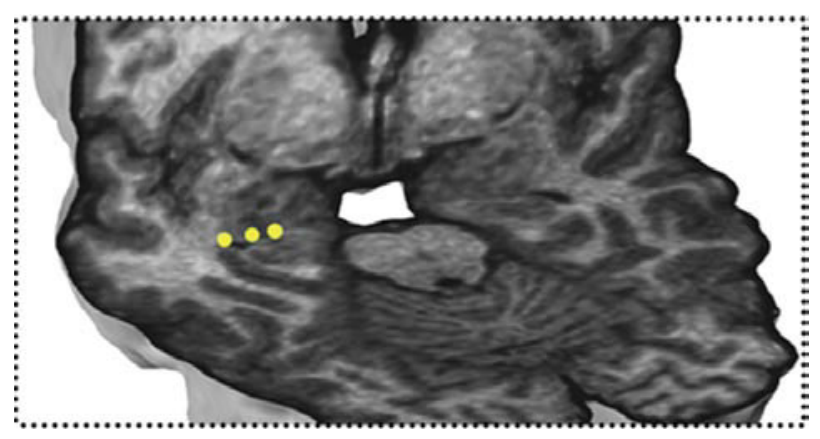

C

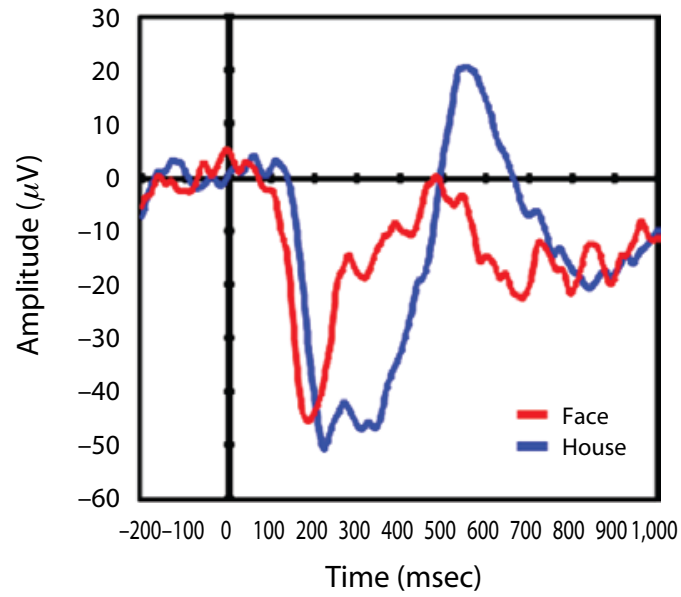

D

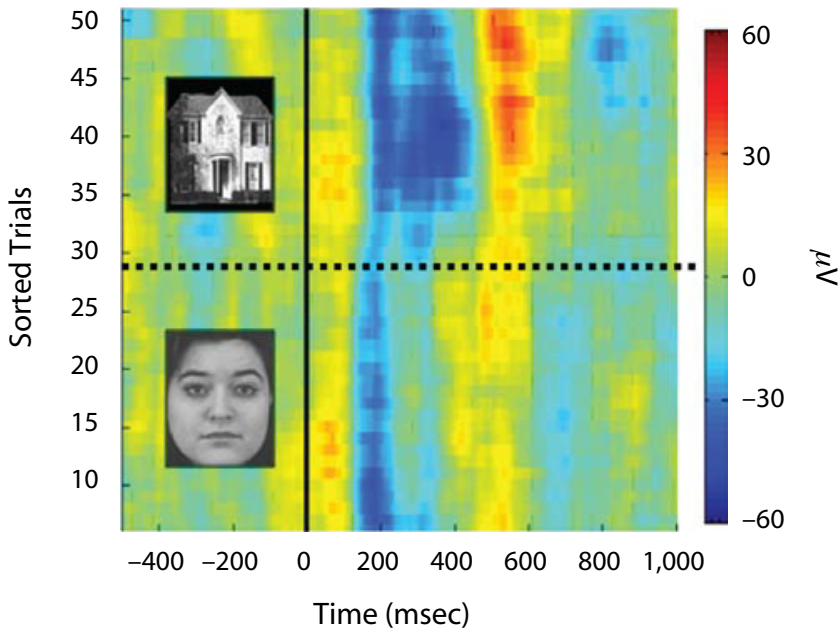

Figure 1. (A) Left back and top view of the patient's brain (T1 MRI reconstructed volume), showing the exact location of the three recorded sites from an intracranial electrode implanted in the left medial temporal lobe and reaching the amygdala, as confirmed by coregistration of MRI and CT scans postimplantation. These three electrodes showed distinctive responses to faces, as compared with houses. Their Talairach coordinates estimated after normalization of brain scans overlapped with the lateral and/or basolateral nucleus of the amygdala (from medial to lateral electrodes: $-22 x,-7 y,-18 z ;-26 x,-6 y,-18 z ;$ and $-31 x,-6 y,-18 z$, respectively) in accordance with results from human imaging studies (Zald, 2003). (B) Mean reaction times (RTs; \pm 1 SEM) obtained in the main attention experiment. Statistical analysis of these behavioral data showed significant main effects of emotion and attention but no interaction, consistent with previous results in this task (Vuilleumier, Armony, Driver, \& Dolan, 2001). (C) Average intracranial amygdala responses (average of all three recording sites) to faces (light trace) and houses (dark trace) as obtained in the independent face localizer experiment. Randomization tests confirmed that responses to faces differed from those to houses during three time windows following stimulus onset (see the Results section), with the earliest difference starting $136 \mathrm{msec}$ post stimulus onset. (D) ERP image (amplitude $\times$ time representation of all consecutive trials; see Delorme \& Makeig, 2004) from the localizer experiment (lateral electrode). Differential responses to faces $(n=28$ trials) and houses $(n=28$ trials) arose during three successive time windows (as confirmed by statistical permutation tests), spanning from 136 to $710 \mathrm{msec}$ postonset, and remained generally stable across the successive trials.

can, \& Lawrence, 2004; Silvert et al., 2007; Vuilleumier et al., 2001; Vuilleumier et al., 2004), in which pictures of fearful or neutral faces were shown together with pictures of houses while attention was directed to either faces or houses. Thus, the task demands were kept unchanged between conditions in which faces were presented at taskrelevant and -irrelevant positions, respectively. On any given trial, neither the relevant stimulus category (face or house) nor the emotional face expression (fearful or neutral) could be predicted, allowing an assessment of purely stimulus-driven responses. This paradigm therefore provides a $2 \times 2$ factorial design allowing us to test for the main effects of emotion versus attention on neural responses to faces, as well as any interaction between these factors. Several fMRI studies using this paradigm in healthy subjects have shown amygdala activation to fearful faces, regardless of whether the faces were task relevant or irrelevant (Bentley et al., 2003; Bishop et al., 
2004; Vuilleumier et al., 2001; Vuilleumier et al., 2004; but see also Silvert et al., 2007). On the basis of these results, we hypothesized that an early $(<200 \mathrm{msec}$ post stimulus onset; see Krolak-Salmon et al., 2004) differential response to fearful versus neutral faces should occur in the amygdala regardless of whether the faces were task relevant or irrelevant. In addition, on the basis of other results suggesting some attentional gating in the amygdala (see Pessoa, McKenna, et al., 2002), we also surmised that some difference might arise at a later latency when face stimuli were task relevant, rather than irrelevant (see Krolak-Salmon et al., 2004; Vuilleumier, 2005).

\section{METHOD}

\section{Case Description}

Patient C.T. was a 30-year-old right-handed male, with a clinical diagnosis of cryptogenic complex partial epilepsy for 15 years. His seizures (frequency: zero to five episodes per month) were characterized by transient losses of consciousness, followed by a postictal decrease of verbal memory performance. A detailed neurological assessment showed no cognitive impairment between seizures, and the patient worked normally as an employee in an international organization. He was treated with a regular antiepileptic drug (leviracetam), which was interrupted after electrode implantation according to a standard weaning protocol. All our investigations were performed during the presurgical evaluation phase in the regular clinical setting, while the patient underwent intracranial EEG with depth electrodes in the left temporal and frontal lobes to identify the epilepsy focus (Seeck et al., 2001; Seeck et al., 1995). A written informed consent was obtained from the patient prior to his participation to this study, following the standard practice in the presurgical evaluation unit (Department of Neurology, University Hospital, Geneva). This research was approved by the local ethics committee. This patient was selected for our experimental protocol on the basis of the following criteria: (1) no interictal cognitive deficit and a normal ability to perform our demanding attention task; (2) at least one electrode implanted in an intact amygdala, with no clinical evidence of structural or epileptic anomalies at the recorded site (Figure 1A); (3) reliable stimulus-driven responses recorded from at least one electrode contact in the amygdala; and (4) no subclinical seizure observed during our intracranial recordings.

\section{Stimuli and Experimental Tasks}

The patient participated in two separate experiments, a localizer task and a main attentional task. The aim of the localizer task was to obtain independent evidence (using a separate set of face stimuli and another task) to demonstrate the selectivity and location of the (left) amygdala responses to faces (relative to houses). Next, the patient performed the main (attention) matching task (Vuilleumier et al., 2001), in which we could examine the processing of fearful faces in face-sensitive contacts of the left amygdala, contrasting two different attentional conditions (in which fearful faces were either task relevant or task irrelevant).

In the two experiments, visual stimuli were shown on a computer screen at a viewing distance of about $1 \mathrm{~m}$, with its center roughly aligned with the level of the eyes. Pictures were presented against a homogeneous dark background and had a similar size across all experiments $(400$ [width] $\times 540$ [height] pixels on a $1,024 \times 768$ resolution screen). The pictures covered approximately $8^{\circ} \times 11^{\circ}$ of visual angle. The average luminance of the stimuli was $\sim 25 \mathrm{~cd} / \mathrm{m}^{2}$.

In the localizer experiment, the patient was shown pictures of a single neutral face or single house with varying viewpoints, presented in a continuous block of 90 stimuli ( 45 per condition, in random order; stimulus duration, $400 \mathrm{msec}$; fixed interstimulus interval [ISI], 3,500 $\mathrm{msec}$ ). The patient was instructed to report any imme- diate repetition of the same stimulus (one-back repetition task; 12 immediate repetitions in total; performance, $99 \%$ correct).

In the main (attention) experiment, the patient viewed brief (750-msec) visual displays comprising two faces (either fearful or neutral) and two houses in vertical and horizontal pairs (see Vuilleumier et al., 2001, for additional details about the stimuli). The position and expression of faces varied pseudorandomly across successive trials. The ISI was kept constant $(3,500 \mathrm{msec})$. The patient fixated a central point and was instructed to concentrate on either the vertical stimulus pairs (first block) or the horizontal stimulus pairs (second block), in order to judge whether the two stimuli at these locations matched or not (i.e., whether the two faces were the same or different, or whether the two houses were the same or different). Orthogonally to this, the expression of the faces was either fearful or neutral, regardless of whether attention was focused on the face pairs (faces relevant) or on the house pairs (faces irrelevant). Each block comprised 96 trials, with the two object categories (faces vs. houses) and their locations fully randomized and counterbalanced across trials, resulting in a total of 24 neutral and 24 fearful faces at task-relevant locations and the same number for each expression at task-irrelevant locations (Vuilleumier et al., 2004).

\section{Intracranial Recordings}

iLFPs were continuously recorded (Ceegraph XL, Biologic System Corps.) with a sampling rate of $512 \mathrm{~Hz}$ (bandpass, 0.1-200 Hz) using several depth electrodes (AD-Tech; electrode diameter, $6 \mathrm{~mm}$; interelectrode spacing, $10 \mathrm{~mm}$ ). LFPs reflect dendritic inputs and processing to a higher degree than does the spiking output of a region (unlike single- or multiunit recordings that are still rarely performed in human patients; but see Fried et al., 1997) but correspond to signals recorded with scalp EEG and correlate with BOLD responses in fMRI (Logothetis et al., 2001). Although the biophysical origin of spiking activity is well understood, less is known about the origin of LFPs. LFPs arise largely from dendritic activity over large brain regions and provide a measure of the input to and local processing within an area. Hence, the LFP constitutes a collective property of a neuronal ensemble (probably spanning several millimeters), and not a property of individual neurons. The primary component measured by the LFP in the cortex is thought to be the excitatory postsynaptic potentials of dendrites, plus after hyperpolarizing potential and afterdepolarizing potential. In other words, the LFP likely reflects subthreshold activity from a large group of surrounding neurons.

The reference electrode was located at position $\mathrm{Cz}$ and the ground at position $\mathrm{FCZ}$ in the 10-20 international EEG system. Intracranial visual evoked potentials were obtained by averaging LFPs timelocked to stimulus onset, for each stimulus category separately. Individual epochs were low-pass filtered using a $30-\mathrm{Hz}$ cutoff. Electrode positions within the brain were determined by a CT scan performed after implantation, coregistered with a high-resolution T1 MRI image, using SPM5 (www.fil.ion.ucl.ac.uk/spm/software/ $\mathrm{spm} 5 /$ ), and then were normalized to define Talairach coordinates of each of the electrode sites (see Figure 1A).

\section{Statistical Analyses of iLFPs}

Single-trial EEG epochs were analyzed offline, after removing all epochs in which propagation of epileptic spikes (from -500 to $+1,000 \mathrm{msec}$ around a stimulus onset) might have spread to the mesial temporal lobe and contaminated the recorded sites ( $\sim 40 \%$, using stringent criteria). In addition, we also eliminated single-trial EEG epochs containing homogeneous epileptic bursts, or high-frequency noise. The number of spike-free trials subsequently used for statistical analyses was roughly similar for the different stimulus conditions, both in the localizer experiment (Figure 1D; faces, 28 trials; houses, 28 trials) and in the main experiment (Figure $2 \mathrm{C}$; fearful faces attended, 28 trials; neutral faces attended, 24 trials; fearful faces unattended, 32 trials; neutral faces unattended, 29 trials). The amplitude variance computed for each time point across these spike-free trials was used as the dependent variable for all statistical comparisons. 
A

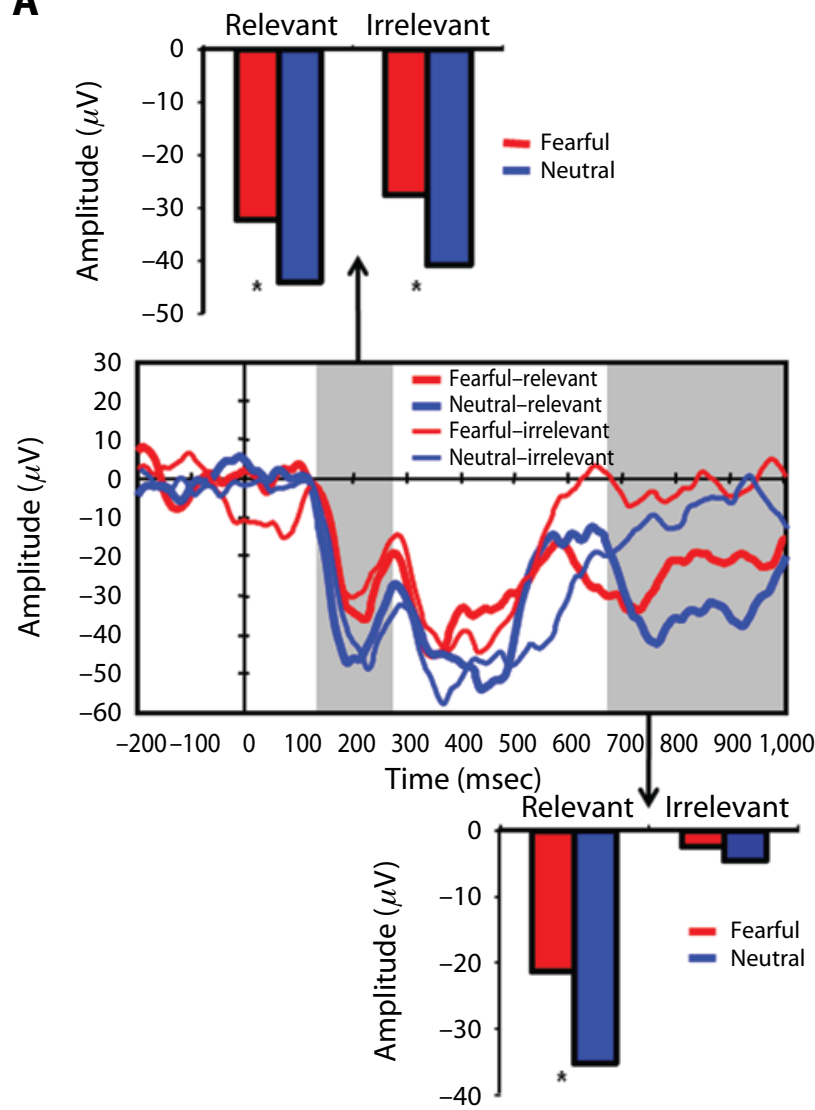

B
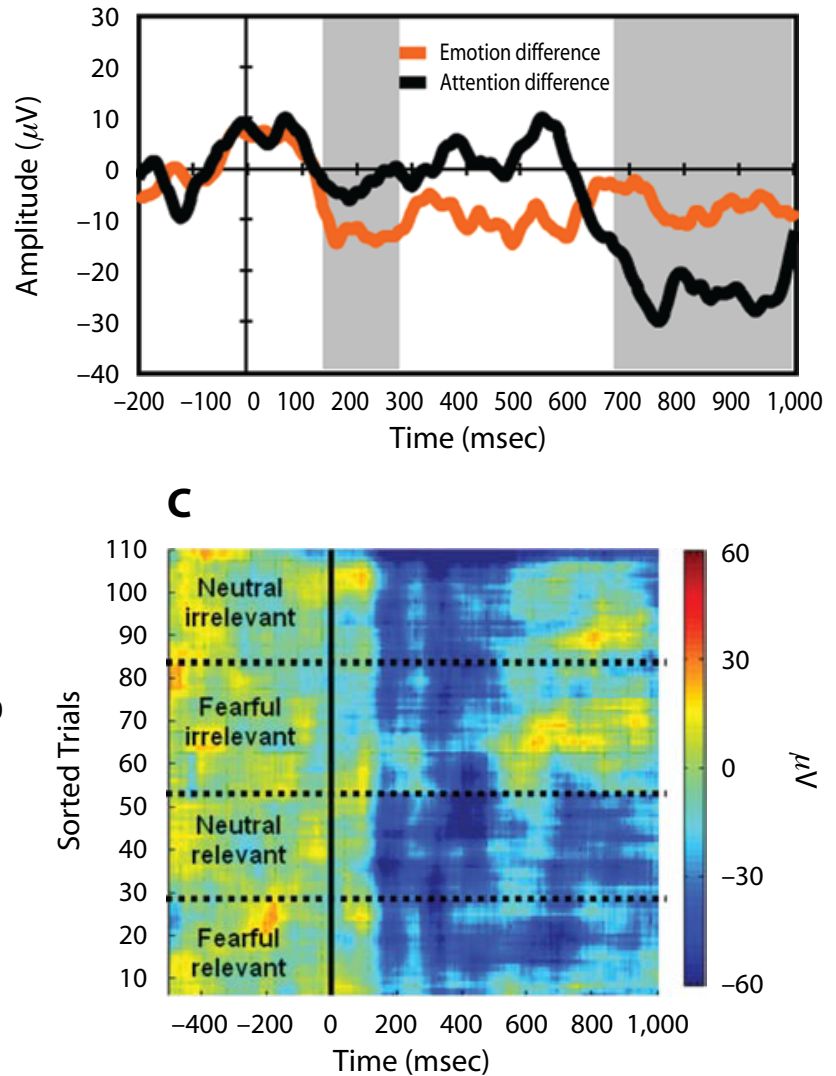

Figure 2. (A) Average local field potentials (all three electrodes collapsed) for each condition in the main experiment, showing responses to fearful faces (light lines) and neutral faces (dark lines) when they were task relevant (thick lines) or task irrelevant (thin lines). A similar time course was observed for fearful faces during the earliest electrical deflection (peaking around $200 \mathrm{msec}$ ), regardless of whether the faces were task relevant or not (see inset above). By contrast, the later negative deflection (peaking between 700 and $800 \mathrm{msec}$ ) was enhanced when faces were task relevant (regardless of expressions) and showed a larger difference between fearful and neutral expressions for task-relevant than for task-irrelevant faces (see inset below), consistent with a delayed gating of emotional responses by attention. For each inset, statistical differences $(p<.05)$ are highlighted by a * symbol. (B) Difference waveforms computed between conditions showing the main effect of emotion (subtraction between neutral and fearful faces; light line) and the main effect of attention (subtraction between task-relevant and task-irrelevant faces; dark line). An emotional modulation was clearly seen from 140 msec onward, whereas the attentional modulation became visible only later, around 710 msec post stimulus onset. (C) ERP image for all the trials in the main experiment (lateral electrode), sorted according to the four different conditions. A biphasic negative deflection occurred from $\sim 120$ to $\sim 500$ msec postonset for all the stimuli ( $n=28$ trials for fearful task relevant, 24 for neutral task relevant, 32 for fearful task irrelevant, and 29 for neutral task irrelevant). Significant amplitude differences (confirmed by statistical permutation tests) were found between emotion conditions (fearful vs. neutral faces) during an early time window (140-290 msec), followed by differences between attention conditions (task-relevant vs. task-irrelevant faces) during a later and longer time window (750-950 msec).

To determine whether amygdala responses were stable over time and across trials, we first computed an amplitude $\times$ time image for all presentations of the stimuli (Delorme \& Makeig, 2004), sorted in the consecutive order of trials for each experimental condition (see Figures $1 \mathrm{D}$ and $2 \mathrm{C}$ ). We also compared the variance (i.e., standard deviation) at each time frame (from -200 to $+1,000 \mathrm{msec}$ around stimulus onset) across the four experimental conditions in order to assess whether this parameter varied or not. An inspection of variance across time points (and conditions) confirmed that it was stable and comparable and that variability or nonlinearity of activity did not create potential drifts in the background signal. These auxiliary analyses therefore confirmed that slight variations in the background signal across single-trial data (see Figures 1D and 2C) could not bias the results in any obvious manner.

The presence of a significant difference between conditions (e.g., face/house or emotion/attention modulations) was verified by nonparametric statistical analyses based on stringent randomization tests (see Manly, 1991, and Pourtois, Peelen, Spinelli, Seeck, \& Vuilleumier, 2007, for recent applications with human intracranial data), allowing reliable inferences for single-subject data. Randomization provides a robust nonparametric statistical method without any assumption regarding data distribution, which compares the observed data set with random shuffling of the same values over many iterations (i.e., permutations). The method runs by repeating the shuffling many times so as to be able to estimate the probability (here, $p<.05$ ) that the data might be observed by chance. In accord with standard procedure used elsewhere (e.g., Pourtois et al., 2007), the significant alpha cutoff was set to $p<.05$, with an additional criterion of temporal stability for five consecutive time points ( $>10 \mathrm{msec}$ at a $512-\mathrm{Hz}$ sampling rate). We used a standard algorithm for permutations applied to the amplitude values of single-trial iLFPs: An empirical distribution of the possible average amplitudes 
was estimated by (1) reassigning single-trial amplitude values to different experimental conditions (i.e., permutations of the data), (2) recalculating the average amplitude, and (3) recalculating the resulting amplitude difference for these "new" average amplitude values. Note that the number of permutations that can be made with a group of $n$ single trials is $2^{n}$, although Manly (1991) suggested that $1,000-5,000$ permutations can be sufficient. Here, we used a systematic number of 5,000 permutations. The mean amplitude value from the actual data was then compared with the values from the empirical distribution to determine the likelihood that the empirical distribution had a value higher than the amplitude value from the actual data. This procedure was then repeated for each time point (time frame).

The onset latencies of negative deflections were determined by computing when iLFP amplitudes at each time frame after stimulus onset differed $(p<.05)$ from baseline values (defined as the mean activity from $-200 \mathrm{msec}$ to stimulus onset). All the statistical analyses of the iLFP data were performed using the Cartool software (developed by Denis Brunet; http://brainmapping.unige.ch/Cartool.php).

To provide another estimate of the reported differences, we also computed the mean amplitude of neural responses for each individual trial in the 140 - to $280-\mathrm{msec}$ time interval poststimulus (corresponding to the emotion effect; see Figure 2A) and submitted these values to a receiver-operating curve (ROC) analysis contrasting fearful to neutral faces (irrespective of whether these faces were task relevant or irrelevant).

\section{RESULTS}

\section{Behavioral Results}

Patient C.T. was highly accurate in all the conditions of the attention task (mean, 95\% correct for face trials, 97\% for house trials), suggesting a balanced task performance for faces and houses.

A conventional ANOVA was performed on single-trial reaction time (RT) data. Although a potential problem for an ANOVA applied to single-subject data is the likelihood of serial correlation (i.e., between successive points), our experimental design minimized the possibility that there would be any serial correlations by using a standard procedure to control for interrelated temporal sequence. First, the order of the presentation of trials in the four conditions was randomized, so as to break up the serial correlation and obtain independence between samples. Second, we allowed ample time intervals between observations (fixed ISI, 3,500 msec), which minimized serial correlations. This analysis showed that the patient was significantly slower to make same/different judgments on faces $(857 \mathrm{msec})$ than on houses $[673 \mathrm{msec}$; main effect of attention condition, $F(1,44)=114.86, p<.001$; see Figure $1 \mathrm{~B}]$ and generally was slower on trials with fearful faces (task relevant, $879 \mathrm{msec}$; task irrelevant, $708 \mathrm{msec}$ ) than on those with neutral faces [task relevant, $835 \mathrm{msec}$; task irrelevant, $639 \mathrm{msec}$; main effect of emotion condition, $F(1,44)=$ $16.17, p<.001$; see Figure 1B]. Thus, this emotion negativity effect (denoting a deeper processing of negative stimuli; see Cacioppo \& Gardner, 1999) arose irrespective of whether the faces were task relevant or not [no attention $x$ emotion interaction; $F(1,44)=1.60, p=.213$ ], replicating previous behavioral results obtained with this task in normal adult healthy subjects (Vuilleumier et al., 2001). We also performed a complementary nonparametric statistical analysis (Friedman test) on the same RT data, which confirmed significant main effects of emotion $(p<.001)$ and of attention $(p<.001)$.

These behavioral results therefore suggest that fearful faces, even when being unattended, led to different emotion processing than did neutral faces. Importantly, differences in RTs between conditions did not account for differences in intracranial ERPs, as was demonstrated by auxiliary analysis on EEG epochs time-locked to motor responses (see below and Figure 3D).

\section{Intracranial Results}

We began by carefully characterizing the electrophysiological properties of amygdala responses to faces, using an independent localizer experiment (neutral face vs. house stimuli). All the statistical analyses were performed using stringent permutation tests. First, we found a typical biphasic negative deflection following stimulus onset (see Krolak-Salmon et al., 2004) that selectively arose at three adjacent electrode sites in the left lateral amygdala (Figure 1C) and whose amplitude started to differ from baseline after $136 \mathrm{msec}$ for faces and $170 \mathrm{msec}$ for houses $(p<$ .01 ; Figure 1C). Auxiliary and exploratory spectral analyses (data not reported here) indicated that this early negative activity corresponded to an event-related perturbation (Delorme \& Makeig, 2004) arising primarily in the theta band, consistent with electrophysiological data on neuronal oscillations in the lateral nucleus of the amygdala in animals ( $\sim 6 \mathrm{~Hz}$; see Pare, 2003). This early activity was followed by more sustained category-related differences, with a lower amplitude of iLFPs for faces than for houses from 230 to $480 \mathrm{msec}(p<.01$; Figure $1 \mathrm{C})$, and then a prolonged negative component to faces from 520 to $710 \mathrm{msec}$ and a positive component to houses during the same period ( $p<$ .01 ; Figure 1C). Reduced amplitude for faces in the latter two time windows was compatible with an involvement of intra-amygdala inhibitory mechanisms after initial excitatory responses (Samson \& Pare, 2006). Additional analyses verified that this activation to faces was stable across successive trials, without significant adaptation over the course of our recording (see Figure 1D) or any significant drift in the background signal (see the Method section).

On the basis of these electrophysiological properties, we predicted that fearful expression and attention to faces should produce a selective modulation of activity at the same amygdala electrodes and within similar time windows during our main experiment (Vuilleumier et al., 2001).

Recordings from face-sensitive sites in the lateral amygdala during the attention task showed an early and systematic differential neural response between fearful and neutral faces, regardless of attention (Figure 2A, upper inset). A statistical comparison (nonparametric permutation test) between the two expressions, pooled across attention conditions, revealed significantly more positive iLFPs from 140 until $290 \mathrm{msec}$ post stimulus onset for fearful than for neutral faces $(p<.01)$, for the same three adjacent electrodes as above. This effect was highly similar for the three recordings sites (see Figures 3A, 3B, and $3 \mathrm{C}$ ). Post hoc comparisons also confirmed that this early emotion effect was significant when faces were either task relevant $(p<.05)$ or task irrelevant $(p<.05)$. 
A

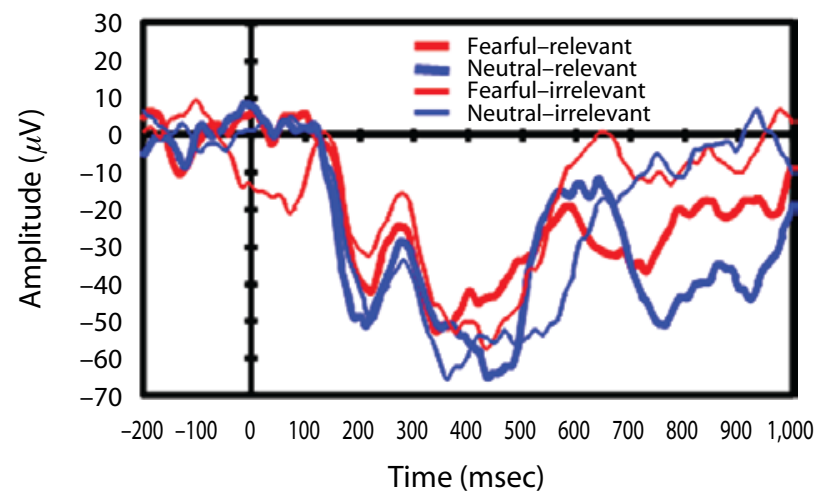

B

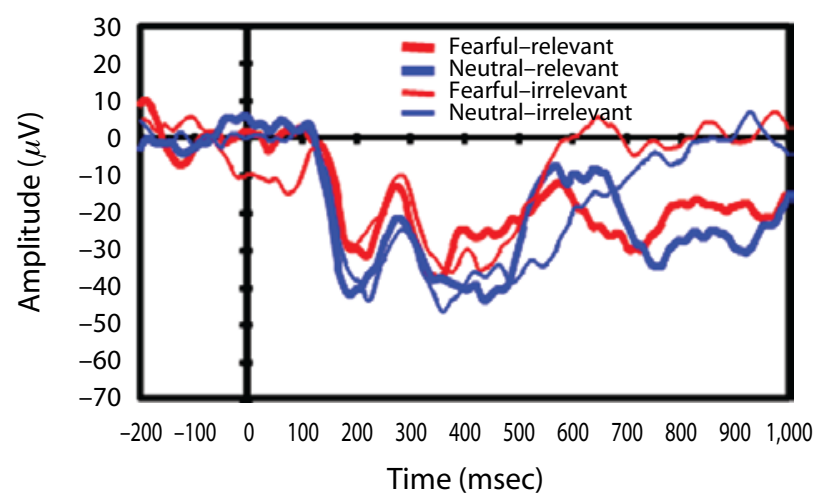

C

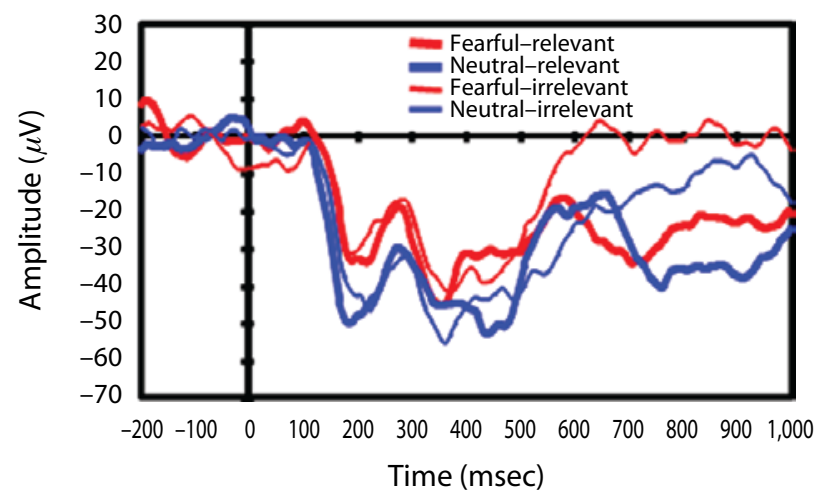

D

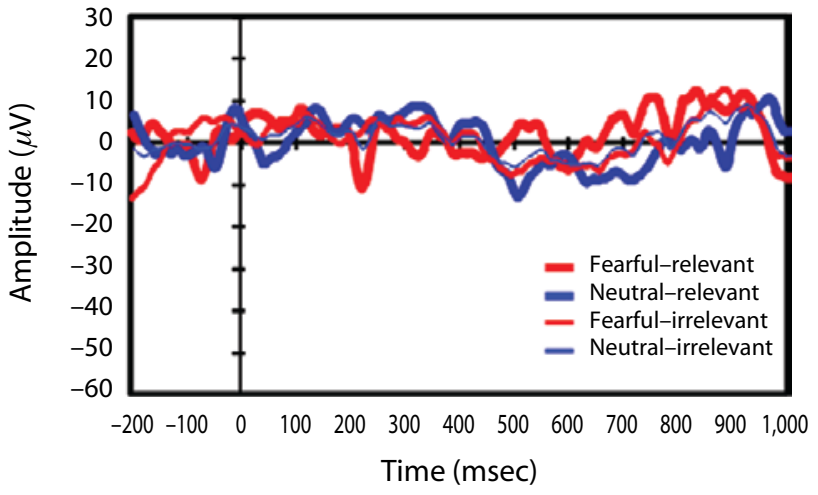

$\mathbf{E}$

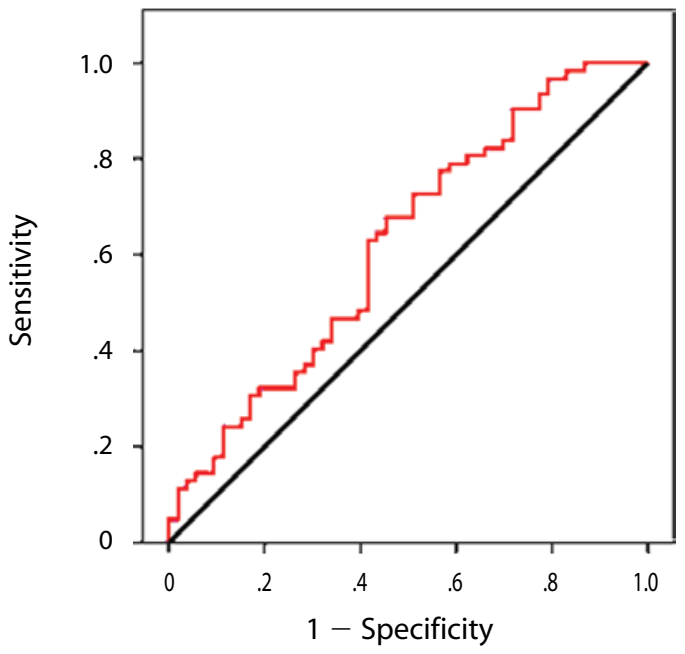

Figure 3. (A) Average local field potentials time-locked to the stimulus onset for each condition in the main experiment shown for the more medial electrode site (A; Talairach coordinates: $-22 x,-7 y,-18 z)$, as well as for $(B)$ the intermediate electrode site (B; Talairach coordinates: $-26 x,-6 y,-18 z)$ and $(C)$ the more lateral electrode site (C; Talairach coordinates: $-31 x,-6 y,-18 z)$, showing consistent responses to fearful faces (light lines) and neutral faces (dark lines) when they were task relevant (thick lines) or task irrelevant (thin lines) at each recording site in the amygdala. (D) Average local field potentials (all three electrodes collapsed) time-locked to the response onset, showing no clear or systematic response-related intracranial ERP components and, thus, indicating that responses in the lateral amygdala after stimulus onset (panels $A, B$, and $C$ ) were not due to decision or motor factors. (E) Receiver operating curve (ROC). We computed the mean amplitude of neural responses for each individual trial in the 140- to 280-msec time interval poststimulus (corresponding to the emotion effect; see Figure $2 \mathrm{~A}$ ) and submitted these values to an ROC analysis contrasting fearful to neutral faces (irrespective of whether they were task relevant or task irrelevant). This analysis confirmed that the amplitudes of neural responses for single-trial data during this time interval were reliably different between fearful and neutral faces (see the Results section). 
Furthermore, we also examined the reliability of the early emotion response with a ROC analysis on the mean iLFP amplitudes recorded for each individual trial during the 140 - to $280-\mathrm{msec}$ time interval post stimulus onset (see the Method section and Figure 3E). The results confirmed that the amplitude of neural responses for single-trial data during this time interval was reliably different between fearful and neutral faces (asymptotic significance, $p=$ .02 ; area under the curve $=.62$ )

Conversely, comparing trials with task-relevant versus task-irrelevant faces (regardless of emotion expression) revealed a sustained attentional effect at the same three face-sensitive electrodes in the left amygdala, but starting at a later time-point $710 \mathrm{msec}$ post stimulus onset $(p<.05)$ and involving more negative potentials for taskrelevant versus task-irrelevant faces (Figure 2A). Computing difference waveforms between conditions clearly demonstrated a differential emotional response that preceded the modulation by task relevance (Figure 2B).

In addition, consistent with an attentional gating of emotional processing in the amygdala (see Pessoa, McKenna, et al., 2002), neural responses during this later time interval (750-950 msec) also interacted with emotional condition: There was a reliable difference in this time window between fearful and neutral faces when they were task relevant $(p<.05)$, but not when they were task irrelevant (Figure 2A, lower inset).

Because these later modulations by attention overlapped with the patient's responses (mean RT $=765 \mathrm{msec}$; see the Behavioral Results section), we also computed intracranial evoked potentials time-locked to response onset (RT). However, this analysis did not reveal any difference between experimental conditions around motor execution (or during the 200-msec preresponse time interval; see Figure 3D). These data thus confirmed that the late LFP modulations were driven by attention or task-related differences in stimulus processing, rather than by motor and decision-related factors or any systematic difference in RTs for face versus house pairs.

\section{DISCUSSION}

By directly recording iLFPs from the left lateral amygdala of a human epileptic patient during a task in which emotion and attention factors were separately manipulated (see Vuilleumier et al., 2001), we could not only test whether attended versus unattended fearful faces elicited a similar differential neurophysiological response, as compared with neutral faces, in the amygdala or not, but also determine the exact latency and duration of this response. On the basis of previous fMRI results (Vuilleumier et al., 2001; Vuilleumier et al., 2004), as well as findings from scalp EEG/MEG recordings during emotion face processing (see Eimer \& Holmes, 2007; Halgren, Raij, Marinkovic, Jousmaki, \& Hari, 2000; Pourtois, Dan, Grandjean, Sander, \& Vuilleumier, 2005; Vuilleumier \& Pourtois, 2007), we hypothesized that an early effect of fearful faces might arise in the amygdala, regardless of whether these were task relevant or irrelevant, and that this might occur with a distinct but later effect of selective attention.
Our new iLFP findings confirmed this assumption and revealed that both task-relevant and task-irrelevant fearful faces elicited an early differential response, as compared with neutral faces, in the lateral amygdala. In the main attention experiment, we found that the amplitude of iLFPs was more positive from 140 - to 290 -msec post stimulus onset for fearful faces, relative to neutral ones $(p<.01)$, irrespective of attention condition, and this emotion effect was equally present for three adjacent electrodes in the amygdala (Figure 3A, 3B, and 3C). The electrophysiological properties and time course of this activation to fearful faces in our patient were very similar to previous intracranial ERP results obtained in the amygdala of four epileptic patients who were also studied during a face perception task after implantation of depth electrodes (see Krolak-Salmon et al., 2004, and Figures 1C and 2A). Our single-trial decompositions (Figures $1 \mathrm{D}$ and $2 \mathrm{C}$ ) verified that this early emotion effect was stable and robust and could be replicated at the single-trial level without any significant habituation of the neural response as a function of time (see Phillips et al., 2001). An additional ROC analysis could further establish that the amplitude of neural responses for single-trial data during this time interval was reliably different between fearful and neutral faces (see Figure 3E). In addition, our recording sites in the lateral amygdala could be characterized as being truly face selective (see also Hoffman et al., 2007; Leonard et al., 1985), based on the results of an independent localizer experiment contrasting neutral faces to houses, which unambiguously revealed an early differential response between these two visual categories with a time course similar to the early emotion effects (Figure 1D). Taken together, these results are in line with previous imaging results suggesting a rapid and relatively "automatic" response to fearful faces in the amygdala, in the sense that it may still arise in conditions in which attention is diverted away from the emotionally significant faces (see Dolan \& Vuilleumier, 2003; Morris et al., 1999; Vuilleumier, 2005).

The direction of amplitude changes for this early emotion effect in the amygdala may appear surprising at first sight, given that neutral faces elicited larger (or more negative) LFPs than did fearful faces. We surmise that this amplitude decrease (or relative shift in polarity) for fearful faces might reflect the activation of selective inhibitory mechanisms that exist between the lateral and the basolateral nucleus of the amygdala and could be recruited early on following stimulus onset, as has previously been shown in animal studies during fear conditioning (Samson \& Pare, 2006). However, it remains difficult to draw definite conclusions from changes in the absolute amplitude (or shifts in the polarity) of intracranial LFPs (as recorded in this study), given that they capture global neurophysiological changes at a macroscopic level (i.e., a neuronal ensemble probably spanning several millimeters). This is unlike animal studies that focus on a much lower level of synaptic changes (measured from single cells) in anatomically distinct nuclei of the amygdala (such as the basolateral and central nucleus; see Samson \& Pare, 2006). Because we could not record neural activity separately from different nuclei of the left amygdala in this study, some caution is needed in the interpreta- 
tion of this amplitude difference reflected by these iLFPs, and no firm conclusion can be drawn from the specific direction of voltage changes between conditions. Thus, the most important and new result of our study is to reveal for the first time a distinct time course in the differential neural activity evoked by emotion and attention within the amygdala nuclei, suggesting separate mechanisms for emotional and attentional effects on face processing in this region. The early emotional effect in the amygdala arose prior to, and independently of, attentional modulation based on current spatial focus. Unlike the early modulation by emotion starting at $140 \mathrm{msec}$ post stimulus onset, the modulation by attention was observed much later, becoming significant only after $700 \mathrm{msec}$ and persisting for more than $300 \mathrm{msec}$ afterward (Figure 2A). Interestingly, our data reveal that this late modulation not only involved a main effect of spatial attention enhancing the neural response to relevant faces, relative to irrelevant faces, but also entailed a selective gating of the processing of fearful expression. Thus, consistent with previous fMRI results (Pessoa, McKenna, et al., 2002), the difference between fearful and neutral faces was detected only when these faces were task relevant but was abolished when these faces were task irrelevant, consistent with a gating of the emotional response in the amygdala. Note that this modulation is much later than the effects of attentional selection on sensory responses in the extrastriate visual cortex (typically starting around 150-200 msec; see Desimone \& Duncan, 1995; Luck, Woodman, \& Vogel, 2000) and might, therefore, correspond to other task-related differences in processing the emotional significance of faces, which would be consistent with a similar late latency (600-800 msec) for intracranial electrical responses of the amygdala to emotional word meaning (see Naccache et al., 2005). Hence, a late attentional gating in the amygdala might potentially be secondary to attention selection taking place in the cortical visual pathways, rather than corresponding to a direct site of attentional selection.

It is noteworthy that we could rule out the alternative account that the late attentional effect in the amygdala was related to overt motor preparation or decision. When we computed intracranial evoked potentials time-locked to response onset (RT; see Figure 3D), we failed to find any difference between experimental conditions around motor execution (or during the 200-msec preresponse time interval; see Figure 3D), despite the substantial overlap between RTs and this late attentional effect (see Figure 1B and the Behavioral Results section). These control analyses therefore corroborated the assumption that the late LFP modulations were driven by attention or task-related differences in stimulus processing, rather than by motor and decision-related factors, or any systematic difference in RTs for face versus house pairs.

There is a wealth of human brain-imaging research showing that amygdala activity may be augmented in some psychiatric disorders, particularly anxiety and depression, and this increase has usually been linked to impaired control exerted by specific regions of the prefrontal cortex, including both ventromedial and dorsolateral prefrontal areas (see Drevets, 2000, 2003). In line with this view, our new results, showing a differential time course of emo- tional versus attentional effects in the amygdala, may suggest that depression (and more generally, mood disorders) affects emotion processing in at least two different ways, implicating the early automatic responses (due to instrinsic changes in amygdala responsivity), the subsequent gating exerted by attention during a later time period (presumably reflecting feedback control from prefrontal regions), or both. It is possible that a reduced gating of emotion by selective attention due to impaired prefrontal activity might contribute to the exacerbation of emotion responses in the amygdala during pathological mood conditions, including (major) depression. Such mechanisms could thus potentially account for the dysregulation of negative affect typically associated with depression, including the inability to disengage attention from negative emotional stimuli (see Koster, De Raedt, Goeleven, Franck, \& Crombez, 2005). Future studies should address this question and test more precisely how depression may affect both the early (emotional) and later (attentional) stages of information processing within the amygdala.

There are several caveats related to the present methodology (single-case study of an epileptic patient) that have to be evoked. First, because we recorded iLFPs from the left amygdala of a single epileptic patient, a straightforward generalization of these statistical results to the (healthy) population was not feasible. Moreover, this patient had a clear epileptic history and, accordingly, was treated with antiepileptic drugs prior to our intracranial testing, which may have also affected normal brain functions. Moreover, because we did not record single-neuron firing rates (see Fried et al., 1997) but iLFPs that primarily reflect dendritic inputs, rather than spiking output (Logothetis et al., 2001), it is possible that some aspects of amygdala processing (e.g., attentional gating) might not be fully captured by the present results. However, LFPs are known to correlate with the fMRI BOLD signal (Logothetis et al., 2001), and thus our results clearly demonstrate a temporal dissociation between emotion and attention effects on amygdala inputs that could not be resolved by previous fMRI studies. Moreover, these effects were recorded from lateral electrodes (Figure 1A), consistent with anatomical data indicating that the lateral nucleus is the main entry of most sensory inputs into the amygdala (Samson \& Pare, 2006).

More generally, our findings show that visual information about facial expression may reach the amygdala rapidly after stimulus onset ( $\sim 140 \mathrm{msec}$; see also Halgren et al., 1994; Krolak-Salmon et al., 2004), a latency similar to that for early emotional responses recorded over anterior scalp regions by surface EEG (Eimer \& Holmes, 2007; Pourtois \& Vuilleumier, 2006), and suggest that some emotional appraisal may take place in the amygdala irrespective of whether stimuli are task relevant or not (see also Vuilleumier et al., 2003), prior to or in parallel with face-specific responses in the visual cortex that typically arise around 150-300 msec (Allison et al., 1994; Allison, Puce, Spencer, \& McCarthy, 1999; Bentin, Allison, Puce, Perez, \& McCarthy, 1996). When comparing these results with our localizer experiment, it is interesting to note that the onset of this early emotional effect (fearful vs. neu- 
tral faces) occurred during the same temporal window as the onset of category-specific effects (faces vs. houses, $136 \mathrm{msec}$; see Figure 1C).

Taken together, the present findings are consistent with separate influences of attention and emotion on stimulus processing (Keil, Moratti, Sabatinelli, Bradley, \& Lang, 2005; Vuilleumier, 2005; Vuilleumier et al., 2001), but also help reconcile discrepant findings of previous fMRI studies. Some studies have reported emotional responses without attentional modulation in the amygdala (e.g., Vuilleumier et al., 2001; Vuilleumier et al., 2004), whereas others have shown significant modulations by attention (e.g., Pessoa, McKenna, et al., 2002). Our new data indicate that both emotion and attention effects take place in the lateral amygdala, but at different latencies. It is possible that different experimental designs (e.g., blocked [see Pessoa, McKenna, et al., 2002] vs. event-related [see Vuilleumier et al., 2001]) might lead to a different sensitivity of fMRI BOLD measures to the early and late phases of amygdala activation. More generally, our findings highlight the importance of tracking the precise time course of neural activity (beyond current fMRI techniques) to a better understanding of brain function.

\section{AUTHOR NOTE}

This work was supported by the National Center of Competence in Research (NCCR) for Affective Sciences, funded by the Swiss National Science Foundation (51NF40-104897) and hosted by the University of Geneva. Thanks to the clinical team of the Presurgical Epilepsy Unit at Geneva Hospital, and to the patient for his collaboration. G.P. was supported by Grant BOF/GOA2006/001 from Ghent University and by a starting grant from the European Research Council (Starting Grant 200758). Correspondence concerning this article should be addressed to G. Pourtois, Department of Experimental Clinical and Health Psychology, Ghent University, Henri Dunantlaan 2, 9000 Gent, Belgium (e-mail: gilles.pourtois@ugent.be).

\section{REFERENCES}

Adolphs, R., Tranel, D., Damasio, H., \& Damasio, A. [R.] (1994). Impaired recognition of emotion in facial expressions following bilateral damage to the human amygdala. Nature, 372, 669-672.

Adolphs, R., Tranel, D., Damasio, H., \& Damasio, A. R. (1995). Fear and the human amygdala. Journal of Neuroscience, 15, 5879-5891.

Allison, T., Ginter, H., McCarthy, G., Nobre, A. C., Puce, A., Luby, M., \& Spencer, D. D. (1994). Face recognition in human extrastriate cortex. Journal of Neurophysiology, 71, 821-825.

Allison, T., Puce, A., Spencer, D. D., \& McCarthy, G. (1999). Electrophysiological studies of human face perception: I. Potentials generated in occipitotemporal cortex by face and nonface stimuli. Cerebral Cortex, 9, 415-430.

Amaral, D. G., Behniea, H., \& Kelly, J. L. (2003). Topographic organization of projections from the amygdala to the visual cortex in the macaque monkey. Neuroscience, 118, 1099-1120.

Anderson, A. K. (2005). Affective influences on the attentional dynamics supporting awareness. Journal of Experimental Psychology: General, 134, 258-281.

Anderson, A. K., Christoff, K., Panitz, D., De Rosa, E., \& GabriELI, J. D. E. (2003). Neural correlates of the automatic processing of threat facial signals. Journal of Neuroscience, 23, 5627-5633.

Anderson, A. K., \& Phelps, E. A. (2001). Lesions of the human amygdala impair enhanced perception of emotionally salient events. Nature, 411, 305-309.

Armony, J. L., \& Dolan, R. J. (2002). Modulation of spatial attention by fear-conditioned stimuli: An event-related fMRI study. Neuropsychologia, 40, 817-826.
Bar, M., Tootell, R. B. H., Schacter, D. L., Greve, D. N., Fischl, B., Mendola, J. D., ET AL. (2001). Cortical mechanisms specific to explicit visual object recognition. Neuron, 29, 529-535.

Bentin, S., Allison, T., Puce, A., Perez, E., \& McCarthy, G. (1996). Electrophysiological studies of face perception in humans. Journal of Cognitive Neuroscience, 8, 551-565.

Bentley, P., Vuilleumier, P., Thiel, C. M., Driver, J., \& Dolan, R. J. (2003). Cholinergic enhancement modulates neural correlates of selective attention and emotional processing. NeuroImage, 20, 58-70.

Bishop, S., Duncan, J., \& Lawrence, A. D. (2004). Prefrontal cortical function and anxiety: Controlling attention to threat-related stimuli. Nature Neuroscience, 7, 184-188.

CACIOPPO, J. T., \& Gardner, W. L. (1999). Emotion. Annual Review of Psychology, 50, 191-214.

Calder, A. J., Lawrence, A. D., \& Young, A. W. (2001). Neuropsychology of fear and loathing. Nature Reviews Neuroscience, 2, 352-363.

Cowey, A., \& Stoerig, P. (1991). The neurobiology of blindsight. Trends in Neurosciences, 14, 140-145.

Critchley, H., Daly, E., Phillips, M., Brammer, M., Bullmore, E., WILliams, S., ET AL. (2000). Explicit and implicit neural mechanisms for processing of social information from facial expressions: A functional magnetic resonance imaging study. Human Brain Mapping, 9, 93-105.

Delorme, A., \& MAKeIG, S. (2004). EEGLAB: An open source toolbox for analysis of single-trial EEG dynamics including independent component analysis. Journal of Neuroscience Methods, 134, 9-21.

Desimone, R., \& Duncan, J. (1995). Neural mechanisms of selective visual attention. Annual Review of Neuroscience, 18, 193-222.

Dolan, R. J., \& Vuilleumier, P. (2003). Amygdala automaticity in emotional processing. In P. Shinnick-Gallagher, A. Pitkanen, A. Shekhar, \& L. Cahill (Eds.), The amygdala in brain function: Basic and clinical approaches (Annals of the New York Academy of Sciences, Vol. 985, 348-355). New York: New York Academy of Sciences.

Drevets, W. C. (2000). Functional anatomical abnormalities in limbic and prefrontal cortical structures in major depression. Progress in Brain Research, 126, 413-431.

Drevets, W. C. (2003). Neuroimaging abnormalities in the amygdala in mood disorders. In P. Shinnick-Gallagher, A. Pitkanen, A. Shekhar, \& L. Cahill (Eds.), The amygdala in brain function: Basic and clinical approaches (Annals of the New York Academy of Sciences, Vol. 985, 420-444). New York: New York Academy of Sciences.

Eimer, M., \& Holmes, A. (2007). Event-related brain potential correlates of emotional face processing. Neuropsychologia, 45, 15-31.

Fried, I., MacDonald, K. A., \& Wilson, C. L. (1997). Single neuron activity in human hippocampus and amygdala during recognition of faces and objects. Neuron, 18, 753-765.

Halgren, E., Baudena, P., Heit, G., Clarke, J. M., Marinkovic, K., \& Clarke, M. (1994). Spatiotemporal stages in face and word processing: I. Depth-recorded potentials in the human occipital, temporal and parietal lobes [corrected]. Journal of Physiology, 88, 1-50.

Halgren, E., RaiJ, T., Marinkovic, K., Jousmaki, V., \& Hari, R. (2000). Cognitive response profile of the human fusiform face area as determined by MEG. Cerebral Cortex, 10, 69-81.

Hoffman, K. L., Gothard, K. M., Schmid, M. C., \& Logothetis, N. K. (2007). Facial-expression and gaze-selective responses in the monkey amygdala. Current Biology, 17, 766-772.

JiANG, Y., \& HE, S. (2006). Cortical responses to invisible faces: Dissociating subsystems for facial-information processing. Current Biology, 16, 2023-2029.

Keil, A., Moratti, S., Sabatinelli, D., Bradley, M. M., \& Lang, P. J. (2005). Additive effects of emotional content and spatial selective attention on electrocortical facilitation. Cerebral Cortex, 15, 1187 1197.

Koster, E. H. W., De Raedt, R., Goeleven, E., Franck, E., \& CromBEZ, G. (2005). Mood-congruent attentional bias in dysphoria: Maintained attention to and impaired disengagement from negative information. Emotion, 5, 446-455.

Kreiman, G., Koch, C., \& Fried, I. (2000). Category-specific visual responses of single neurons in the human medial temporal lobe. Nature Neuroscience, 3, 946-953. 
Krolak-Salmon, P., Henaff, M. A., Vighetto, A., Bertrand, O., \& MAUGUiERE, F. (2004). Early amygdala reaction to fear spreading in occipital, temporal, and frontal cortex: A depth electrode ERP study in human. Neuron, 42, 665-676.

LeDoux, J. (1996). The emotional brain: The mysterious underpinnings of emotional life. New York: Simon \& Schuster.

Leonard, C. M., Rolls, E. T., Wilson, F. A., \& Baylis, G. C. (1985). Neurons in the amygdala of the monkey with responses selective for faces. Behavioural Brain Research, 15, 159-176.

Logothetis, N. K., Pauls, J., Augath, M., Trinath, T., \& OelterMANN, A. (2001). Neurophysiological investigation of the basis of the fMRI signal. Nature, 412, 150-157.

Luck, S. J., Woodman, G. F., \& Vogel, E. K. (2000). Event-related potential studies of attention. Trends in Cognitive Sciences, 4, 432-440.

Manly, B. F. (1991). Randomization and Monte Carlo methods in biology. London: Chapman \& Hall.

Morris, J. S., DeGelder, B., Weiskrantz, L., \& Dolan, R. J. (2001). Differential extrageniculostriate and amygdala responses to presentation of emotional faces in a cortically blind field. Brain, 124, 12411252.

Morris, J. S., Frith, C. D., Perrett, D. I., Rowland, D., Young, A. W., Calder, A. J., \& Dolan, R.-J. (1996). A differential neural response in the human amygdala to fearful and happy facial expressions. Nature, 383, 812-815.

Morris, J. S., Ohman, A., \& Dolan, R. J. (1999). A subcortical pathway to the right amygdala mediating "unseen" fear. Proceedings of the National Academy of Sciences, 96, 1680-1685.

Naccache, L., Gaillard, R., Adam, C., Hasboun, D., Clemenceau, S., BAUlaC, M., ET AL. (2005). A direct intracranial record of emotions evoked by subliminal words. Proceedings of the National Academy of Sciences, 102, 7713-7717.

Nakamura, K., Mikami, A., \& Kubota, K. (1992). Activity of single neurons in the monkey amygdala during performance of a visual discrimination task. Journal of Neurophysiology, 67, 1447-1463.

Ohman, A., \& MineKa, S. (2001). Fears, phobias, and preparedness: Toward an evolved module of fear and fear learning. Psychological Review, 108, 483-522.

PARE, D. (2003). Role of the basolateral amygdala in memory consolidation. Progress in Neurobiology, 70, 409-420.

Pasley, B. N., Mayes, L. C., \& Schultz, R. T. (2004). Subcortical discrimination of unperceived objects during binocular rivalry. Neuron, 42, 163-172.

Pessoa, L., Japee, S., Sturman, D., \& Ungerleider, L. G. (2006). Target visibility and visual awareness modulate amygdala responses to fearful faces. Cerebral Cortex, 16, 366-375.

Pessoa, L., Kastner, S., \& Ungerleider, L. G. (2002). Attentional control of the processing of neural and emotional stimuli. Cognitive Brain Research, 15, 31-45.

Pessoa, L., McKenna, M., Gutierrez, E., \& Ungerleider, L. G. (2002). Neural processing of emotional faces requires attention. Proceedings of the National Academy of Sciences, 99, 11458-11463.

Pessoa, L., Padmala, S., \& Morland, T. (2005). Fate of unattended fearful faces in the amygdala is determined by both attentional resources and cognitive modulation. NeuroImage, 28, 249-255.

Phelps, E. A. (2006). Emotion and cognition: Insights from studies of the human amygdala. Annual Review of Psychology, 57, 27-53.

Phelps, E. A., \& LeDoux, J. E. (2005). Contributions of the amygdala to emotion processing: From animal models to human behavior. $\mathrm{Neu}$ ron, 48, 175-187.

Phillips, M. L., Medford, N., Young, A. W., Williams, L., Williams, S. C. R., Bullmore, E. T., ET AL. (2001). Time courses of left and right amygdalar responses to fearful facial expressions. Human Brain Mapping, 12, 193-202.

Pourtois, G., Dan, E. S., Grandjean, D., Sander, D., \& VuiLLEUMIER, P. (2005). Enhanced extrastriate visual response to bandpass spatial frequency filtered fearful faces: Time course and topographic evoked-potentials mapping. Human Brain Mapping, 26, 65-79.

Pourtois, G., Peelen, M. V., Spinelli, L., Seeck, M., \& VuiLLEUMIER, P. (2007). Direct intracranial recording of body-selective responses in human extrastriate visual cortex. Neuropsychologia, $\mathbf{4 5}$, 2621-2625.
Pourtois, G., Schwartz, S., Seghier, M. L., Lazeyras, F., \& VuiLLEUMier, P. (2006). Neural systems for orienting attention to the location of threat signals: An event-related fMRI study. NeuroImage, 31, 920-933.

Pourtois, G., \& Vuilleumier, P. (2006). Dynamics of emotional effects on spatial attention in the human visual cortex. Progress in Brain Research, 156, 67-91.

Sabatinelli, D., Bradley, M. M., Fitzsimmons, J. R., \& Lang, P. J. (2005). Parallel amygdala and inferotemporal activation reflect emotional intensity and fear relevance. NeuroImage, 24, 1265-1270.

SAMsON, R. D., \& PARE, D. (2006). A spatially structured network of inhibitory and excitatory connections directs impulse traffic within the lateral amygdala. Neuroscience, 141, 1599-1609.

Seeck, M., Michel, C. M., Blanke, O., Thut, G., Landis, T., \& Schomer, D. L. (2001). Intracranial neurophysiological correlates related to the processing of faces. Epilepsy \& Behavior, 2, 545557.

Seeck, M., Schomer, D., Mainwaring, N., Ives, J., Dubuisson, D., Blume, H., ET AL. (1995). Selectively distributed processing of visual object recognition in the temporal and frontal lobes of the human brain. Annals of Neurology, 37, 538-545.

Silvert, L., Lepsien, J., Fragopanagos, N., Goolsby, B., Kiss, M., TAYLOR, J. G., ET AL. (2007). Influence of attentional demands on the processing of emotional facial expressions in the amygdala. NeuroImage, 38, 357-366.

Surguladze, S. A., Brammer, M. J., Young, A. W., Andrew, C., Travis, M. J., Williams, S. C. R., \& Phillips, M. L. (2003). A preferential increase in the extrastriate response to signals of danger. NeuroImage, 19, 1317-1328.

VUilleumier, P. (2005). How brains beware: Neural mechanisms of emotional attention. Trends in Cognitive Sciences, 9, 585-594.

Vuilleumier, P., Armony, J. L., Driver, J., \& Dolan, R. J. (2001). Effects of attention and emotion on face processing in the human brain: An event-related fMRI study. Neuron, 30, 829-841.

Vuilleumier, P., Armony, J. L., Driver, J., \& Dolan, R. J. (2003). Distinct spatial frequency sensitivities for processing faces and emotional expressions. Nature Neurosciences, 6, 624-631.

Vuilleumier, P., \& Driver, J. (2007). Modulation of visual processing by attention and emotion: Windows on causal interactions between human brain regions. Philosophical Transactions of the Royal Society $B, \mathbf{3 6 2}, 837-855$.

Vuilleumier, P., \& Pourtois, G. (2007). Distributed and interactive brain mechanisms during emotion face perception: Evidence from functional neuroimaging. Neuropsychologia, 45, 174-194.

Vuilleumier, P., Richardson, M. P., Armony, J. L., Driver, J., \& Dolan, R. J. (2004). Distant influences of amygdala lesion on visual cortical activation during emotional face processing. Nature Neuroscience, 7, 1271-1278.

Vuilleumier, P., \& Schwartz, S. (2001). Beware and be aware: Capture of spatial attention by fear-related stimuli in neglect. NeuroReport, 12, 1119-1122.

Whalen, P. J., Kagan, J., Cook, R. G., Davis, F. C., Kim, H., Polis, S., ET AL. (2004). Human amygdala responsivity to masked fearful eye whites. Science, 306, 2061.

Whalen, P. J., Rauch, S. L., Etcoff, N. L., McInerney, S. C., Lee, M. B., \& JENIKE, M. A. (1998). Masked presentations of emotional facial expressions modulate amygdala activity without explicit knowledge. Journal of Neuroscience, 18, 411-418.

Williams, M. A., McGlone, F., Аввотt, D. F., \& Mattingley, J. B. (2005). Differential amygdala responses to happy and fearful facial expressions depend on selective attention. NeuroImage, 24, 417-425.

Williams, M. A., Morris, A. P., McGlone, F., Авbott, D. F., \& MatTINGLEY, J. B. (2004). Amygdala responses to fearful and happy facial expressions under conditions of binocular suppression. Journal of Neuroscience, 24, 2898-2904.

ZALD, D. H. (2003). The human amygdala and the emotional evaluation of sensory stimuli. Brain Research Reviews, 41, 88-123.

(Manuscript received March 11, 2009; revision accepted for publication August 16, 2009.) 